\title{
FIRST EVIDENCE FOR THE EXISTENCE OF INSTANTONS IN THE QUANTIZED SU(2) LATTICE VACUUM
}

\author{
E.-M. ILGENFRITZ ${ }^{1}$, M.L. LAURSEN ${ }^{2}$ *, M. MÜLLER-PREUBKER ${ }^{3}$, \\ G. SCHIERHOLZ $Z^{2,4}$ and H. SCHILLER \\ ${ }^{\prime}$ Sektion Physik, Karl-Marx-Universität, Leipzig, GDR \\ 'Deutsches Elektronen-Synchrotron DESY, Hamburg, FRG \\ ${ }^{3}$ Sektion Physik, Humboldt-Universität, Berlin, GDR \\ 'Institut für Theoretische Physik der Universität, Kiel, FRG \\ ${ }^{5}$ Institut für Hochenergiephysik, A kademie der Wissenschaften, Zeuthen, GDR
}

Received 18 October 1985

Starting from Monte Carlo generated equilibrium gauge field configurations at $\beta=2.1$ and 2.2 on a $6^{4}$ lattice, representing the physical vacuum, we systematically freeze the quantum fluctuations by means of successive relaxations. The result is that we obtain (approximate) solutions of the classical equations of motion, which turn out to have discrete values of the action

$$
S \approx \beta 2 \pi^{2} N, \quad N=0,1,2, \ldots
$$

in close agreement with the continuum (multi-) instanton solutions. We show that these "lattice (multi-) instantons" are localized in space-time, that they carry a topological charge $|Q|=N$ and that they give rise to a number of fermion zero modes in accordance with the Atiyah-Singer index theorem. Finally, we estimate the "background topological susceptibility" from the distribution of lattice (multi-) instantons.

\section{Introduction}

To "solve" QCD it is not enough to compute the mass spectrum, condensates, the topological susceptibility, etc. by lattice Monte Carlo methods [1]. It is equally important to get a qualitative understanding of confinement, chiral symmetry breaking and the like - i.e. of the QCD vacuum.

A necessary and sufficient condition for confinement is the existence of random domains of colour magnetic flux [2]. One would think that the energy it costs to patch up such a vacuum - thinking of the vortices as being necessary to do so - outweights the energy that is gained in "growing" the domains. In QCD there is, however, the possibility of the formation of instantons [3] - i.e. semi-classical,

^ Address after 1 September 1985: NORDITA, Copenhagen, Denmark. 
dipole-like objects of minimal action with unit topological charge - which could amalgamate the random domains with little cost in energy.

Though, in this picture, instantons may not be the "driving force" of confinement, we believe that they play a key role in the physics of the QCD vacuum. This belief receives further support from the observation that, semi-classically, instantons may provide a resolution of the $\mathrm{U}(1)$ problem [4] as well as a mechanism for chiral symmetry breaking [5].

So far the question whether the QCD vacuum does possess an underlying instanton structure, has only found an indirect answer: it has been shown by lattice Monte Carlo calculations that the vacuum of the SU(2) gauge theory is topologically non-trivial and that the topological susceptibility is of the right order of magnitude as required for a quantitative resolution of the $U(1)$ problem $[6,7]$. The early semi-classical calculations, on the other hand, have remained of uncertain validity in spite of great efforts undertaken during the last years [8] to cure its infrared problems.

In the present paper we shall try to find a more direct answer to this question. The idea is to generate equilibrium lattice gauge field configurations, i.e. "snapshots" of the physical vacuum, and subsequently freeze their quantum fluctuations by successive relaxations. If there are instantons underlying the vacuum, they should remain behind and become visible, as they are quasi-stable under relaxations, i.e. (approximate) solutions of the classical equations of motion. A similar procedure has been applied previously to the $O(3)$ sigma model [9].

The paper is organized as follows. In sect. 2 we describe the procedure of relaxation and ask the question, to what extent it leads to reproducible results. In sect. 3 we collect evidence that the resulting, quasi-stable field configurations represent (multi-) instantons. In sect. 4 we estimate the background topological susceptibility and in sect. 5 we draw our conclusion.

\section{Relaxation of lattice gauge field configurations}

We start from equilibrium SU(2) gauge field configurations, which we have generated by standard Monte Carlo methods using the Wilson action

$$
S=\beta \sum_{n, \mu<\nu}\left(1-\frac{1}{2} \operatorname{Tr} U_{n, \mu \nu}\right)=\beta \sum_{n, \mu<\nu}\left[1-\frac{1}{2} \operatorname{Tr}\left(U_{n, \mu} U_{n+\mu, \nu} U_{n+\nu, \mu}^{+} U_{n, \nu}^{+}\right)\right]
$$

Now we replace successively each link matrix, $U_{n, \mu}$ by

$$
U_{n, \mu} \rightarrow \bar{U}_{n, \mu}=c \sum_{\substack{\nu \\(\mu<\nu)}}\left[U_{n, \nu} U_{n+\nu, \mu} U_{n+\mu, \nu}^{+}+U_{n-\nu, \nu}^{+} U_{n-\nu, \mu} U_{n+\mu-\nu, \nu}\right]
$$




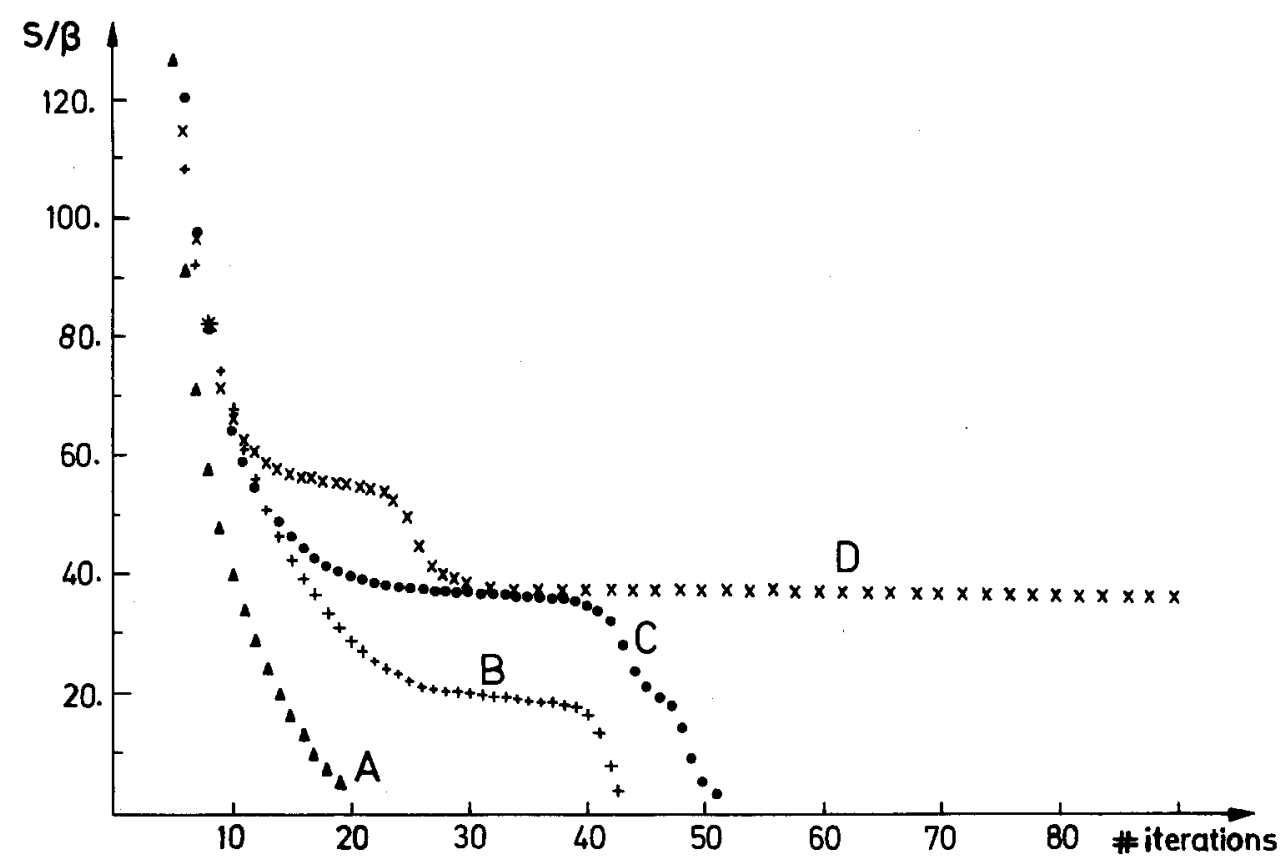

Fig. 1. The ratio $S / \beta$ as a function of the number of iterations for 4 typical gauge field configurations at $\beta=2$.1. The first iterations with $S / \beta \geq 120$ are not shown.

where $c$ is a normalization constant such that

$$
\bar{U}_{n, \mu} \in \mathrm{SU}(2)
$$

When all link matrices have been changed once, we call this one iteration. This procedure will locally minimize the action. One can also think of other methods of "cooling" the gauge field configurations. A similar algorithm can also be given for $\mathrm{SU}(3)[10]$.

Our sample of vacuum configurations consist of 40 configurations at $\beta=2.1$ and 18 configurations at $\beta=2.2$ on a $6^{4}$ lattice. In fig. 1 we have shown the history of 4 typical gauge field configurations under successive relaxation. While configuration $A$ decays into the trivial $(S=0)$ vacuum, configurations $\mathrm{B}, \mathrm{C}$ and $\mathrm{D}$ show a plateau, indicating the passage through a quasi-stable field configuration. On the plateaus the action takes the values

$$
S=\beta\left(2 \pi^{2}-\Delta\right) N, \quad N=1,2, \ldots,
$$

with $\Delta \simeq 1$. This is in close agreement with what one would expect for a continuum (multi-) instanton field configuration, i.e. $S=\beta 2 \pi^{2} N$. The fact that the lattice action 
TABLE 1

The correlation matrix $M_{N \bar{N}}$ as defined in the text

\begin{tabular}{l|r|rrrrrrr}
\hline \multicolumn{7}{c}{ reverse ordering } \\
\hline
\end{tabular}

(4) is somewhat smaller than the continuum value is presumably due to the finite volume of the lattice [11].

In the following we shall call a configuration quasi-stable if after one iteration it changes its action by less than 2 . The fact that the relaxation procedure employed here does not lead to absolutely stable lattice field configurations has already been observed in ref. [12]. We disagree, however, with the authors' conclusion that the Wilson action does not support the existence of instantons.

If the observed quasi-stable lattice field configurations are an attribute of the equilibrium configuration they came from, their appearance should, by and large, not depend on the particular relaxation procedure used. One way of checking this is to apply a different algorithm to compute the new link matrix $\bar{U}_{n, \mu}$ from the old ones, e.g. by using the Langevin equation without noise or a modified Metropolis algorithm. In this paper we have performed a different test: we have "cooled" our sample of 40 configurations at $\beta=2.1$, in addition, by sweeping through the lattice in the reverse order. To compare the outcome of the two procedures we have computed in table 1 the correlation matrix

$$
M_{N \bar{N}}=40(N \bar{N})^{-1}\langle N \bar{N}\rangle,
$$

where $N, \bar{N}$ is the height of the first (quasi-stable) plateau in units of $S / \beta\left(2 \pi^{2}-\Delta\right)$ for the "forward" and the reverse ordering, respectively. We find that the two procedures give in 17 out of 35 (identifiable) cases the same answer. In order to underpin the statistical significance of this result we calculate

$$
\tau=\sum_{N, \bar{N}} 40(N \bar{N})^{-1} \frac{(\langle N \bar{N}\rangle-\langle N\rangle\langle\bar{N}\rangle)^{2}}{\langle N\rangle\langle\bar{N}\rangle},
$$




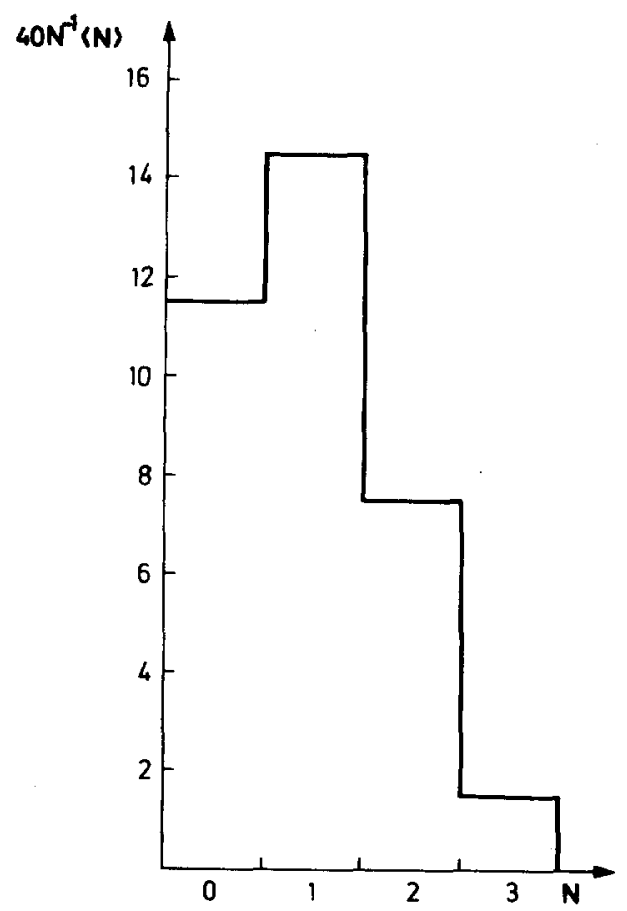

Fig. 2. The frequency $40 N^{-1}\langle N\rangle$ of finding a quasi-stable configuration with $N=S / \beta\left(2 \pi^{2}-\Delta\right)$ for the configurations of table 1 .

which gives $\tau=31.9$. For a $\chi^{2}$ distribution for 16 degrees of freedom there is only a $1 \%$ probability that $\chi^{2}>\tau$, so that the possibility for both sets of values being statistically independent is extremely small. To check the stability of the results further, we have "cooled" the field configuration which gave $N, \bar{N}=3$ (and is relatively infrequent) a further 10 times by sweeping through the lattice in sequential order but starting from randomly chosen links. In all cases we found a $N=3$ plateau. All together we take this as (statistical) evidence for a real underlying semi-classical field structure already at $\beta=2.1$.

In fig. 2 we have plotted the frequency $40 N^{-1}\langle N\rangle$ of finding a quasi-stable, $N=S / \beta\left(2 \pi^{2}-\Delta\right)$ field configuration among the configurations of table 1 , where we have averaged the results for forward and reverse ordering.

\section{Do the quasi-stable configurations support the picture of an instanton?}

Before we can interpret the quasi-stable lattice configuration obtained in the last section as instantons, we must demonstrate that their action density is localized in space-time, that they are solutions of the classical (lattice) equations of motion, that 
they have the right topological charge and that they give rise to the right number of fermion zero modes.

\subsection{ACTION DENSITY}

During the process of relaxation we have monitored the action density,

$$
S_{n}=\frac{1}{6} \beta \sum_{\mu<\nu}\left(1-\frac{1}{2} \operatorname{Tr} U_{n, \mu \nu}\right)
$$

As an example we have plotted in fig. 3a-c $p_{n}=\left[100 S_{n} / \beta\right]$ for configuration B of fig. 1 after 30 successive iterations, where the dots indicate $p_{n}=0$. The time slices not shown have action densities $p_{n}=0$ everywhere. This shows that the action density is indeed localized. Similar plots for configurations C and D (on the plateaus) show that the action is concentrated in 2 and 3 well separated lumps which resembles the picture of a dilute instanton gas.

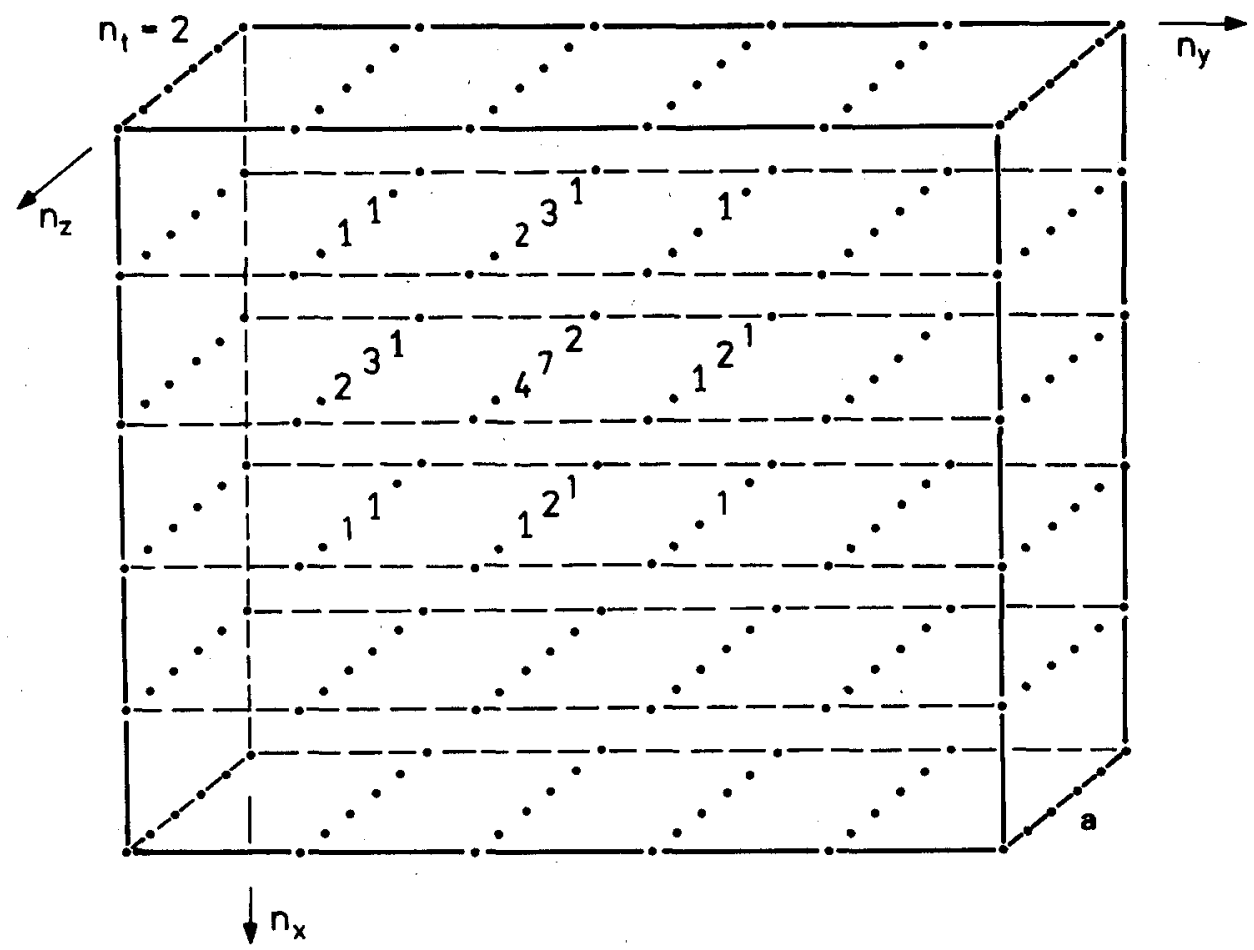

Fig. 3. The action density $S_{n}$ of configuration B (fig. 1) after 30 iterations. The integers plotted are $p_{n}=\left[100 S_{n} / \beta\right]$. The dots correspond to $p_{n}=0$. 

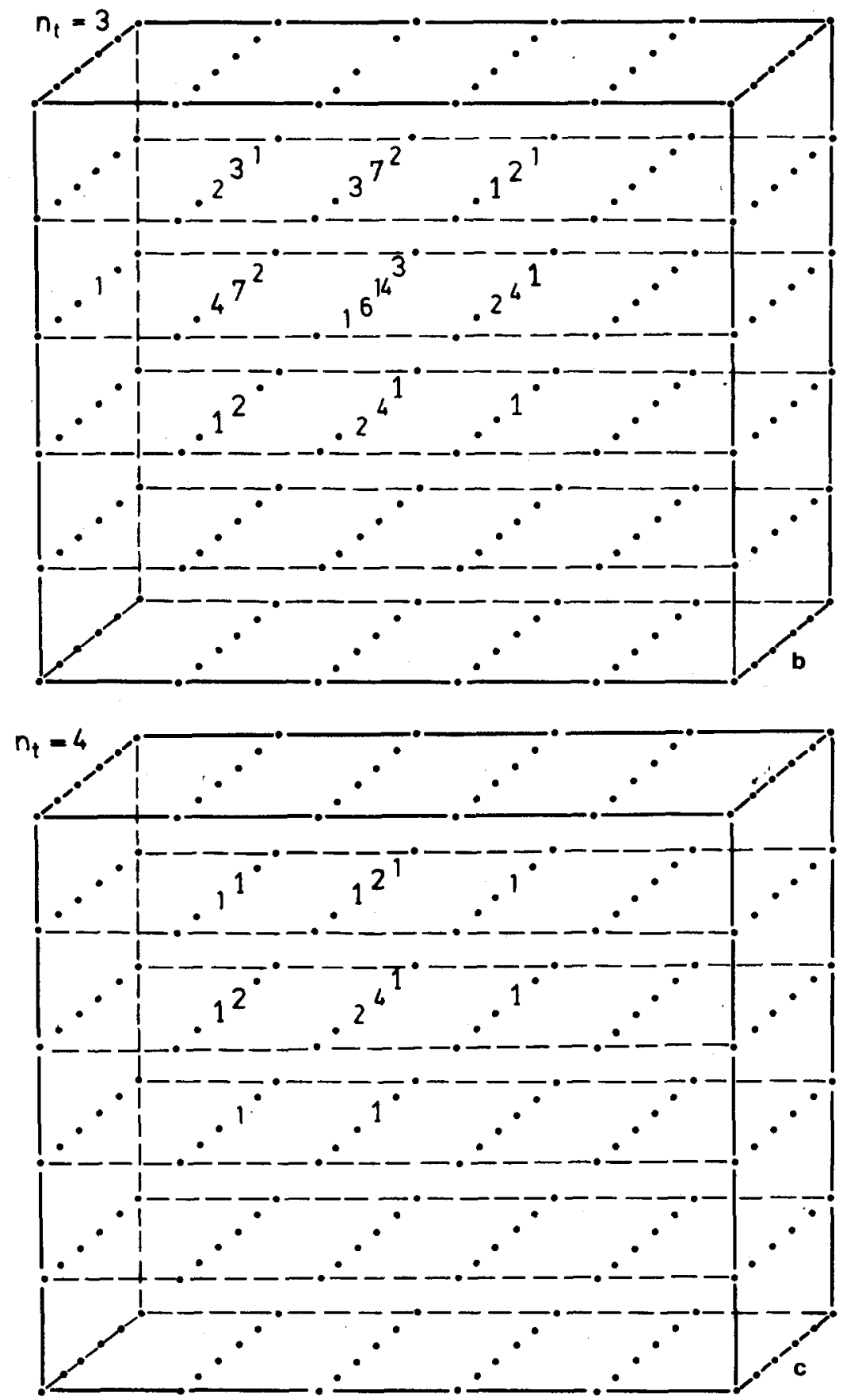

Fig. 3 (continued). 


\subsection{LATTICE EQUATIONS OF MOTION}

The relaxation procedure does not guarantee that the resulting field configurations represent (always) a solution of the classical lattice field equations

$$
\frac{\delta S}{\delta U_{n, \mu}}=0
$$

i.e.

$$
\sum_{\nu}\left(U_{n, \mu \nu}-U_{n, \mu \nu}^{+}\right)=0
$$

To find out to what extent the quasi-stable configurations are solutions of the classical equations of motion, we define the local deviation of the actual link matrix $U_{n, \mu}$ from its replacement value $\bar{U}_{n, \mu}$ (cf. eq. (2)):

$$
\delta_{n}=\frac{1}{2} \sum_{\mu} \operatorname{Tr}\left[\left(U_{n, \mu}-\bar{U}_{n, \mu}\right)\left(U_{n, \mu}-U_{n, \mu}\right)^{+}\right] .
$$

We have calculated $\delta_{n}$ for all lattice sites after each iteration. We find that when we get down to the plateau, $\delta_{n}$ becomes small for all $n$ and remains so for typically the first half of the plateau. In the second half $\delta_{n}$ then starts locally to increase and, as we approach the region where the action turns into another plateau or to zero, it develops a sharp peak at exactly the maximum (or one of the maxima) of $S_{n}$ - which also becomes more pronounced as we move along. The "instanton" thus shrinks, becomes a dislocation [13] and finally is annihilated.

\subsection{TOPOLOGICAL CHARGE}

We have computed the topological charge $Q$ of the quasi-stable field configurations using Lüscher's definition $[6,14]$ of $Q$. On the plateaus - more precisely, throughout the whole plateaus until the configurations collapse, the secondary plateaus included - we found for all configurations

$$
Q= \pm N= \pm S / \beta\left(2 \pi^{2}-\Delta\right)
$$

(despite the fact that Lüscher's bound [14], $S_{n} / \beta<0.015$, is locally violated; cf. fig. $3 a-c$ where $S_{n} / \beta \leq 0.14$ ). This is exactly what we expect for a (multi-) instanton field configuration. We shall call this charge the background charge.

In fig. 4 we have plotted the background charge distribution of our sample of 18 equilibrium gauge field configurations at $\beta=2.2$. The background charge distribution of the 40 configurations at $\beta=2.1$ can be read off from fig. 2 (for $|Q|$ though). 


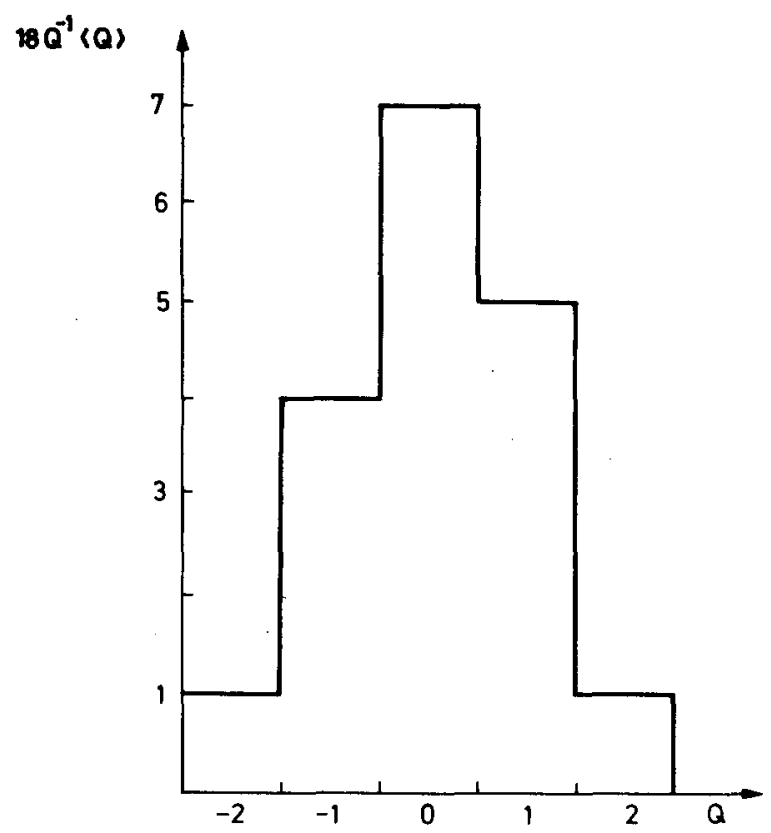

Fig. 4. Distribution of the background topological charge for the sample of configurations at $\beta=2.2$.

\subsection{FERMION ZERO MODES}

The lattice action for massless Kogut-Susskind fermions in a background field configuration $\left\{U_{n, \mu}\right\}$ is given by

$$
S_{\mathrm{F}} \equiv \bar{\chi} M(U) \chi=\sum_{n, \mu}\left[\bar{\chi}_{n}(-1)^{n_{1}+\cdots+n_{\mu-1}} U_{n, \mu} \chi_{n+\mu}-\text { h.c. }\right]
$$

where $\bar{\chi}_{n}, \chi_{n}$ are single component, colour doublet Grassmann variables sitting at the sites $n\left(=\left(n_{1}, n_{2}, n_{3}, n_{4}\right)\right)$. We assume antiperiodic boundary conditions in all 4 directions. $S_{\mathrm{F}}$ then has an explicit chiral symmetry for which $\langle\bar{\chi} \chi\rangle$ is an order parameter. In the infinite (lattice) volume limit we have [15]

$$
\langle\bar{\chi} x\rangle=2 \pi\langle\rho(0)\rangle,
$$

where $\rho(\lambda)$ is the density of eigenvalues $\{\lambda\}$ of the fermion matrix $i M(U)$. It is thus the existence of zero modes that will determine whether chiral symmetry is broken spontaneously or not.

In the continuum the Atiyah-Singer index theorem [16] states that the number of zero modes, $Z$, of self-dual or anti-self-dual gauge fields is equal to $|Q|$. To check this we have computed the eigenvalues of the fermion matrix associated with the quasi-stable field configurations using the Lanczos algorithm developed in ref. [15]. 
On a finite lattice we will, of course, not find exact zero modes but (at most) small eigenvalues which are well separated from the rest of them. On the plateaus we found

$$
Z=|Q|=N
$$

in accordance with the index theorem. To illustrate this, we have plotted $\rho(\lambda)$ for 3 typical field configurations in fig. 5a-c. Configuration $E$, which has
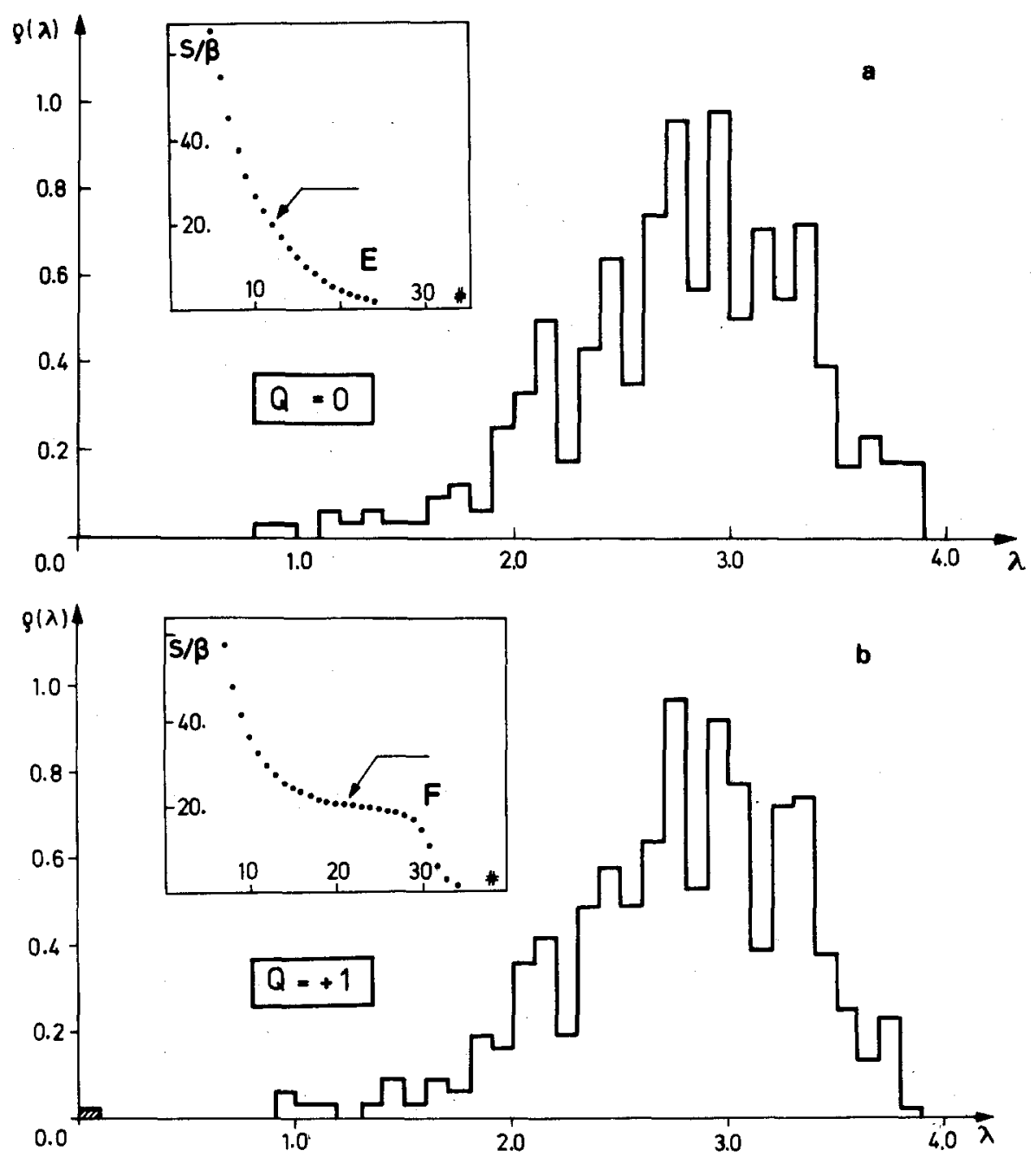

Fig. 5. (a)-(c) The eigenvalue density $\rho(\lambda)$ as a function of $\lambda$ for 3 typical gauge field configurations at $\beta=2.2$. The insert shows the corresponding $S / \beta$ as a function of the number of iterations, and the arrow indicates where $\rho(\lambda)$ and $Q$ were computed. (d) The eigenvalue density $\rho(\lambda)$ for a typical equilibrium configuration. 

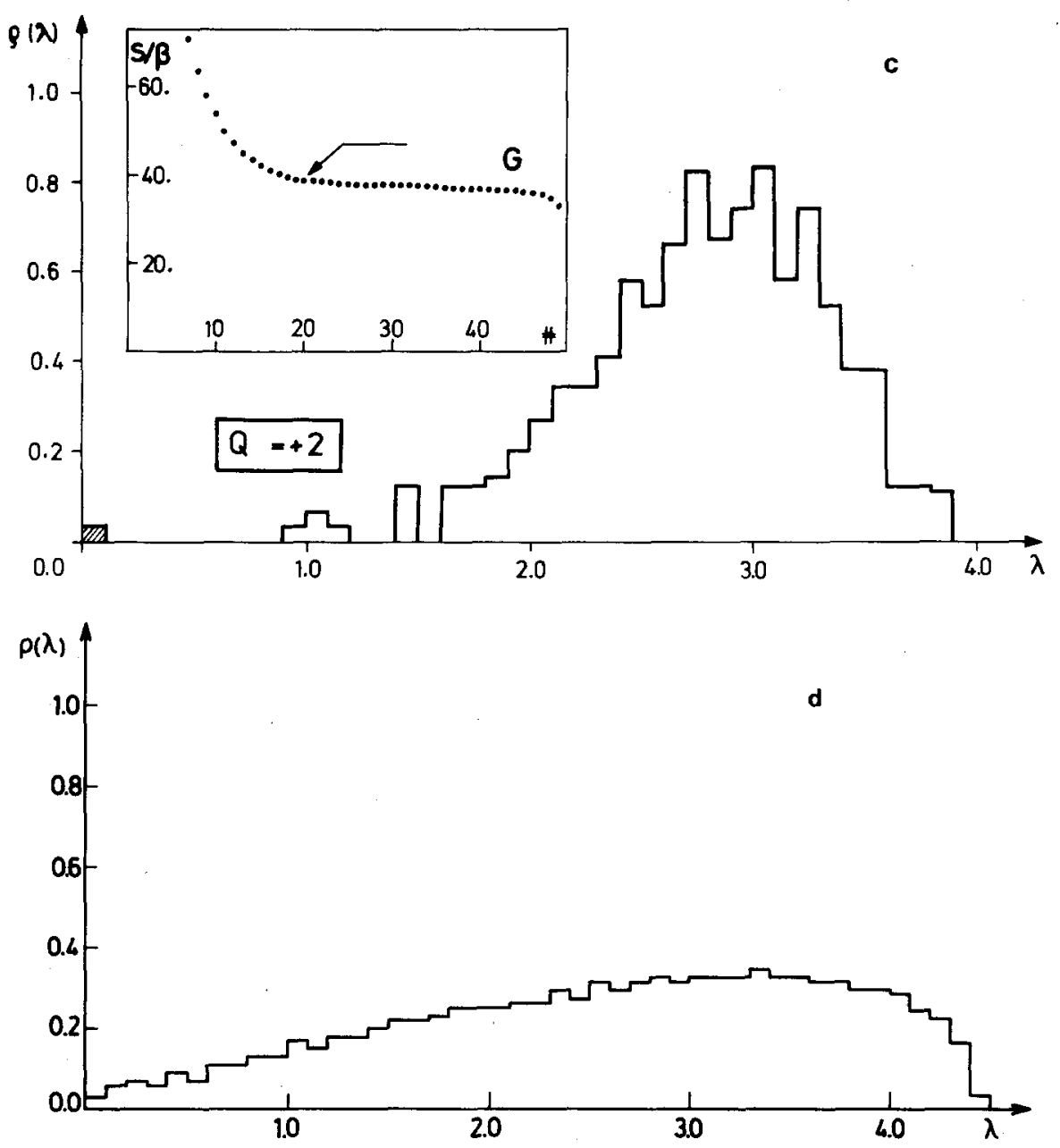

Fig. 5 (continued).

$Q=0$, has also no "zero" mode. Configuration $\mathrm{F}$, which has $Q=1$, has one "zero" mode (not counting the degeneracy of the eigenvalues due to the fermion doubling), while configuration $\mathrm{G}$, which has $Q=2$, supports exactly 2 "zero" modes.

For comparison we have also shown $\rho(\lambda)$ for a typical equilibrium configuration (that of fig. 5b) in fig. 5d. The difference in the two eigenvalue spectra is striking: while $\rho(\lambda)$ extends continuously to $\lambda \rightarrow 0$ for the equilibrium configuration, it develops a gap in the process of relaxation leaving behind the "zero" modes.

To conclude this section, we may say that the quasi-stable field configurations underlying the equilibrium configurations have passed all tests so that they can be interpreted as (multi-) instantons. It would be interesting now to compare these 
configurations with an analytic expression for an instanton field configuration. The construction of such an expression on the lattice is made difficult, however, because it has to be periodic in all 4 directions. In ref. [17] a construction of a lattice instanton was given, which had the right topological charge and exactly one "zero" mode. The basic idea was to map $\mathbb{R}^{4}$ onto a finite lattice, which was divided into an inner and outer region. In the inner (outer) region the gauge fields were taken to be the discretized instanton solution of the continuum equations of motion in the regular (singular) gauge, and in the overlap region they were patched together by a gauge transformation. Unfortunately, we find that this construction violates the classical equations of motion in the overlap region, and therefore it collapses under relaxation.

\section{Estimate of the background topological susceptibility}

In ref. [6] the topological susceptibility, $\chi_{\mathrm{t}}=\left\langle Q^{2}\right\rangle / V$, was found to be

$$
\chi_{\mathrm{t}}=\left(40.8 \pm 1.3 \Lambda_{\mathrm{L}}\right)^{4}
$$

for values of $\beta$ ranging between 2.2 and 2.5 , which, taking [6] $\Lambda_{\mathrm{L}} \approx 6 \mathrm{MeV}$, gives

$$
\chi_{\mathrm{t}}=(245 \pm 8 \mathrm{MeV})^{4}
$$

The question now is: can this be attributed to instantons?

To answer this question, we have computed the topological charge of the equilibrium configurations and compared it to the corresponding background charge. We find that the equilibrium and the background charges are not strongly correlated. A similar test as that described in sect. 2 gives (for 32 degrees of freedom) a $80 \%$ probability that the two sets of charges are statistically independent. For the background topological susceptibility we obtain

$$
\begin{aligned}
& \beta=2.1: \quad \chi_{\mathrm{t}} \approx\left(20 \Lambda_{\mathrm{L}}\right)^{4}=(120 \mathrm{MeV})^{4}, \\
& \beta=2.2: \quad \chi_{\mathrm{t}} \approx\left(21.7 \Lambda_{\mathrm{L}}\right)^{4}=(130 \mathrm{MeV})^{4},
\end{aligned}
$$

which is one order of magnitude below the value (15), (16).

This is, maybe, not surprising. It is possible that the $0^{-}$glueballs contribute to $\chi_{\mathrm{t}}$, which would be on the quantum level. But it could also be that at the present values of $\beta$ the gauge fields are not smooth enough to allow for an unambiguous determination of the topological charge. 


\section{Conclusion}

We have shown that even on small lattices and for values of $\beta$ at the edge of the continuum region the vacuum of the quantized (pure) $S U(2)$ gauge theory does possess an underlying instanton structure.

The lattice size (and hence the range of $\beta$ values) was dictated to us by the fact that the computation of $Q[6,14]$ is very time consuming. However, we are now in the possession of an algebraic expression for the topological charge on the lattice [18], which is fast to compute. This will allow us to repeat the investigation on larger lattices and for larger values of $\beta$, which will be the subject of a future report.

\section{Note added in proof}

After this work had been completed we learnt that M. Teper [19] has done a similar investigation to ours.

\section{References}

[1] J. Kripfganz, talk at XXII. Int. Conf. on high energy Physics, Leipzig. 1984;

G. Schierholz, talk at 27th Scottish Universities Summer School in Physics, St. Andrews, 1984 , CERN preprint TH-4139/85, to be published in the proceedings

[2] P. Olesen, Nucl. Phys. B200 (1982) 381

[3] A.A. Belavin, A.M. Polyakov, A.S. Schwartz and Yu.S. Tyupkin, Phys. Lett. 59B (1975) 85

[4] G. 't Hooft, Phys. Rev. Lett. 37 (1976) 8; Phys. Rev. D14 (1976) 3432

[5] C. Callan, R. Dashen and D. Gross, Phys. Rev. D17 (1978) 2717

[6] I.A. Fox, J.P. Gilchrist, M.L. Laursen and G. Schierholz, Phys. Rev. Lett. 54 (1985) 749

[7] Y. Arian and P. Woit, Stony Brook preprint ITP-SB-85-60 (1985)

[8] E.-M. Ilgenfritz and M. Müller-Preußker: Nucl. Phys. B184 (1981) 443; Phys. Lett. 99B (1981) 128;

E.V. Shuryak, Nucl. Phys. B203 (1982) 93, 116, 140;

G. Münster, Z. Phys. C12 (1982) 43;

D.I. Dyakonov and V.Yu. Petrov, Nucl. Phys. B245 (1984) 259

[9] B. Berg, Phys. Lett. 104B (1981) 475;

Y. Iwasaki and T. Yoshié, Phys. Lett. 127B (1983) 197

[10] J. Hoek, Rutherford preprint RAL-85-042 (1985)

[11] $M$. Lüscher, private communication

[12] Y. Iwasaki and T. Yoshié, Phys. Lett. 131B (1983) 159

[13] B. Berg, ref. [9];

B. Berg and M. Lüscher, Nucl. Phys. B190 (1981) 412;

M. Lüscher, Nucl. Phys. B200 [FS4] (1982) 61

[14] M. Lüscher, Comm. Math. Phys. 85 (1982) 39

[15] I.M. Barbour, P. Gibbs, J.P. Gilchrist, G. Schierholz, H. Schneider and M. Teper, Phys. Lett. 136B (1984) 80

[16] M. Atiyah and I. Singer, Ann. Math. 87 (1968) 484

[17] I.A. Fox, J.P. Gilchrist, M. Göckeler, M.L. Laursen and G. Schierholz, Phys. Lett. 158B (1985) 332

[18] M. Göckeler, M.L. Laursen, G. Schierholz and U.-J. Wiese: DESY preprint 85-142 (1985);

A. Phillips and D. Stone, Stony Brook preprint

[19] M. Teper, CERN preprint TH-4208 (1985) 